\title{
L'argument linguistique chez Cheikh Anta Diop et ses disciples
}

publié dans Fauvelle-Aymar F.-X., Chrétien J.-P. et Perrot C.-H. (éds.), 2000, Afrocentrismes : l'histoire des Africains entre Egypte et Amérique, Paris, Karthala, pp. 79-102.

\author{
Henry Tourneux \\ Langues et Cultures d'Afrique noire
}

CNRS

Il faut reconnaître à C.A. Diop le mérite d'avoir rappelé à beaucoup que l'Egypte fait partie du continent africain et que les barrières que les manuels scolaires de notre enfance dressaient autour de cette région n'ont pas lieu d'être. Cela dit, ce renversement de perspective l'a entraîné, avec dans sa suite, quelques disciples qui ont voulu développer son héritage, à vouloir faire de l'Egypte le centre qui explique tout en Afrique ${ }^{1}$. Estimant prouvée l'unité culturelle de l'Afrique noire et de l'Egypte ancienne - 1'Egypte ancienne faisant partie de l'Afrique noire -, l'unité linguistique est postulée, puis « démontrée ».

\section{Importance de l'argument linguistique}

On ne peut manquer d'être frappé, quand on lit l'oeuvre de Cheikh Anta Diop, par l'abondance des études linguistiques qu'elle contient. Nous ne citerons que les plus importantes, figurant dans des ouvrages :

1. Nations nègres et culture: pp. 191-470, 485-529

2. L'Afrique noire précoloniale : pp. 223-266

3. Antériorité des civilisations nègres : pp. 43-64, 108-114

4. Parenté génétique de l'égyptien pharaonique : XXXVII + 402 p.

5. Nouvelles recherches sur l'égyptien ancien, $221 \mathrm{p}$.

Alors que C.A. Diop limitait son étude à un face à face wolof-égyptien (englobant marginalement le sereer, le sara, le diola, etc.), ses successeurs ont tenté d'élargir les perspectives à d'autres langues : duala (Dika Akwa), $m b o s i$, puis l'ensemble des langues négro-africaines, exception faite des langues khoisan, de l'éthio-sémitique et du berbère (Obenga), pulaar (Lam), etc.

${ }^{1}$ Il arrive cependant que quelqu'un se pose une question embarrassante : l'araire étant bien connue en Egypte, et même représentée par un hiéroglyphe, comment se fait-il qu'elle ne se retrouve nulle part en Afrique noire? Lam 1993, p. 217. 
Les études linguistiques de C.A. Diop et T. Obenga sont toutes caractérisées par une bibliographie affligeante ${ }^{2}$ dans le domaine négro-africain. A.M. Lam, se cantonnant au pulaar, cerne mieux son sujet de ce point de vue.

\section{Qu'est-ce que la linguistique historique africaine?}

C.A. Diop suggérait modestement en $1977^{3}$ qu'il fondait peut-être réellement la linguistique historique africaine grâce à son étude sur la parenté génétique de l'égyptien pharaonique et des langues négro-africaines. M. Houis, dès 1980, tout en évitant de se prononcer sur la thèse centrale de l'auteur - à savoir la parenté entre l'égyptien ancien et le wolof -, sans non plus dénier la légitimité d'une recherche sur ce point, mettait en évidence le caractère inapproprié de la présentation des faits wolof ${ }^{4}$ par C.A. Diop. Il contestait également la validité de la méthode employée pour la démonstration :

«Diop suit [...] la tradition philologique en prenant les données sans les situer rigoureusement dans les systèmes de relations où se trouvent justifiés leurs valeurs et leurs statuts. [...] Nous pensons qu'une telle option [...] a aujourd'hui quelque chose d'anachronique [...]. $»^{5}$

Une quinzaine d'année plus tard, T. Obenga pense lui aussi fonder une linguistique historique en Afrique ${ }^{6}$. La linguistique historique africaine, suivant cet auteur, qui se situe dans la lignée de C.A. Diop et reprend sa thématique, a pour objectif de montrer «l'unité linguistique génétique de l'égyptien pharaonique, du copte et des langues négro-africaines modernes ». Cette unité est acquise depuis qu'elle a été pratiquement ratifiée par des archéologues au fameux colloque égyptologique organisé par l'Unesco au Caire en 19747. D'après cet auteur, tant que l'on n'acceptera pas ce fait, aucun travail sérieux ne pourra se faire en linguistique africaine.

«Pour avoir méconnu ou écarté la langue égyptienne de son champ de recherche fondamentale, la linguistique générale n'a pas encore retrouvé les origines des langues négro-africaines, leur communauté prédialectale, leur profondeur historique. Il est aisé de constater en effet qu'aucune classification des langues négro-africaines tentée à ce jour ne comporte une quelconque temporalité. »

Précisons dès maintenant qu'il n'est pas du ressort de la classification d'établir une chronologie.

\footnotetext{
${ }^{2} C f$. Bouquiaux 1996.

${ }^{3}$ Diop 1977, p. XXIV.

${ }^{4}$ Houis, 1980, pp. 73-78.

${ }^{5}$ Ibid. pp. 71-72.

${ }^{6}$ Obenga 1993, pp. 7-8.

${ }^{7}$ Obenga 1998, p. 60.
} 


\section{Le thème du complot et de la falsification}

Un thème récurrent chez Cheikh Anta Diop est celui du complot. Les Modernes sont coupables

«[...] d'avoir occulté, tant aux yeux des Africains que des Européens, l'existence de véritables civilisations en Afrique précoloniale et l'antériorité de sa plus ancienne civilisation : l'Egypte. D'avoir refusé d'en attribuer le mérite à des hommes de couleur noire. $»^{8}$.

Cet auteur, généralement, réserve les attaques de ce genre aux historiens.

T. Obenga étend la liste des «coupables» aux linguistes, qui ne sont généralement pas cités nommément. Ainsi,

«Le chamito-sémitique est un mythe dans le cadre strict de la science linguistique. $»^{9}$

«[...] l'écran linguistique 'chamito-sémitique' ou 'afro-asiatique' a été inventé de toutes pièces, justement pour 'couper' culturellement la Vallée du Nil égypto-nubienne du reste de l'Afrique noire. $»^{10}$

«On abuse gravement de la bonne foi du profane en disant que le pluriel sémitique concorde avec le pluriel égyptien [...]. $»^{11}$

A.M. Lam ${ }^{12}$ ne se sent pas victime du même complot, puisque, parmi les linguistes qui se sont intéressés anciennement à la langue peule (appelée pulaar au Sénégal), il y en a eu à revendiquer une origine égyptienne pour celle-ci. Il cite dans cette ligne Lilias Homburger à qui il rend hommage, tout en soulignant sa faible compétence en pulaar, qui l'expose à de sérieuses erreurs ${ }^{13}$, sans toutefois invalider ses résultats, apparemment.

\section{Classification et méthode comparative}

Joseph H. Greenberg (1966) a révolutionné les classifications des langues africaines très cloisonnées que l'on avait établies avant lui, démontrant l'indépendance du fait linguistique de toutes considérations relatives au nombre de locuteurs d'une langue ou d'un groupe de langues donnés, à leur aspect physique ou à leur mode de vie.

\footnotetext{
${ }^{8}$ Cf. Fauvelle 1996, pp.73-118.

${ }^{9}$ Obenga 1993, p. 82.

${ }^{10}$ Ibid. p. 10.

${ }^{11}$ Ibid. p. 89.

${ }^{12}$ Lam 1993.

${ }^{13}$ Ibid. p. 296.
} 
Ainsi, il a intégré les centaines de langues bantoues dans une grande famille où se retrouvent la majeure partie des langues d'Afrique de l'Ouest, leur attribuant dans la classification la même place que si elles n'avaient constitué qu'une seule langue. Il a réuni dans un même phylum, appelé "Afroasiatique » le sémitique, l'ancien égyptien, le berbère, le couchitique et le tchadique. Il a démantelé la pseudo-famille hamitique de Meinhof, qui reposait sur des fondements mythiques, raciaux et typologiques. Sur ce point, il est en parfait accord avec C.A. Diop qui écrivait :

«On est incapable de faire correspondre à la notion de Chamite [...] la moindre réalité historique, géographique, linguistique ou ethnique. $»^{14}$

On peut dire qu'en cette fin de vingtième siècle, aucun linguiste ne remet en cause les grandes lignes de cette classification, même si des aménagements $\mathrm{y}$ ont été apportés ${ }^{15}$. Une nouvelle famille a été créée au sein de l'Afroasiatique $^{16}$, un regroupement a été proposé entre le Congo-Kordofanien (appelé actuellement "Niger-Kordofanien ») et le Nilo-Saharien ${ }^{17}$, la place du songhay, du mandé, du hadza est encore discutée. Peu importe, le cadre général est parfaitement valide.

M. Ruhlen explique bien comment l'on classe les langues :

«En fait, on découvre des relations entre les langues non pas en effectuant des comparaisons directes, par exemple entre le français et le chinois, mais plutôt en classant les langues en familles, celles-ci en familles plus grandes, et ainsi de suite $[\ldots]^{18}$. »

Ensuite, l'identification d'un groupe de langues comme constituant une famille valide « résulte à chaque fois, sans aucune exception, de l'examen du vocabulaire de base $[\ldots]^{19}$. »

La classification permet d'inférer la parenté des langues regroupées sur la base de ressemblances de couples forme / sens :

«Dire que plusieurs langues appartiennent à un même groupe, c'est dire qu'elles sont des différenciations d'une langue plus ancienne: des

\footnotetext{
${ }^{14}$ Diop 1965, p. 37.

${ }^{15}$ Voir, entre autres, Newman 1995, Boyd 1996, Ruhlen 1997.

${ }^{16}$ Il s'agit de l'Omotique, qui a été retiré du Couchitique par plusieurs spécialistes. Voir, par exemple, Bender L. (éd.) 1976, The Non-Semitic Languages of Ethiopia, African Studies Center, Michigan State University, Michigan, pp. 298-438.

${ }^{17}$ Voir Gregersen 1972, Boyd 1996.

${ }^{18}$ Ruhlen 1997, p. 15.

${ }^{19}$ Ibid., p. 232.
} 
langues parentes sont en réalité une seule et même langue modifiée de manières diverses au cours du temps. $»^{20}$

La procédure de classification est entièrement distincte de celle que propose la méthode comparative, qui a été développée dans le domaine de l'indoeuropéen. Cette dernière se propose de reconstruire, à partir des faits relevés dans une famille, certains éléments de la langue commune dont sont issus les membres de la famille. Elle présuppose l'établissement d'une parenté par une méthode de classification ${ }^{21}$.

La méthode comparative a été appliquée avec succès aux domaines bantou, voltaïque, tchadique, etc. Elle implique l'établissement de correspondances phonétiques régulières entre les sons présumés de la langue commune et ceux des langues qui en dérivent. Plus la distance temporelle entre langue commune et langue-fille est grande, plus il est difficile, généralement d'établir ce type de correspondances.

\section{Position de C.A. Diop, T. Obenga et A.M. Lam par rapport à J.H. Greenberg}

Cheikh Anta Diop ignore superbement l'oeuvre de Greenberg, dont il ne pouvait pourtant pas ne pas avoir entendu parler, puisque C. Tardits a publié dès 1955 une traduction de ses travaux sur la classification des langues africaines dans le Bulletin de l'IFAN22.

A.M. Lam, qui connaît cette version ancienne des travaux de Greenberg, ne semble pas en prendre ombrage; du moins la classification proposée par Greenberg ne lui pose-t-elle pas de questions particulières, centré qu'il est sur la question peule. En outre, sa thèse centrale, qui revendique la négritude des Peuls, est confortée par la classification de leur langue au sein du Congo-Kordofanien.

T. Obenga, lui, range J.H. Greenberg parmi les savants ambigus, qui ont, d'un côté, «créé de toutes pièces »le «mythe chamito-sémitique $»^{23}$, ce qui est la supercherie majeure de la linguistique; d'un autre côté, il salue en Greenberg celui

«qui a beaucoup contribué à l'avancement de la linguistique générale en Afrique, en établissant des liens étroits, substantiels, entre les langues

\footnotetext{
${ }^{20}$ Meillet 1975, p. 78.

21 Ruhlen 1997 rappelle à juste titre que la reconnaissance de la famille indoeuropéenne a précédé la phase de reconstruction. La reconstruction n'est pas une preuve de la parenté, mais elle ne peut être menée à bien que s'il y a parenté.

${ }^{22}$ Bulletin de l'IFAN 1955, série $\mathrm{B}, \mathrm{T}$. XVI, $\mathrm{n}^{\circ} 1-2$ et T. XVII, $\mathrm{n}^{\circ}$ 1-2.

${ }^{23}$ Obenga 1993, p. 79.
} 
bantu et les langues de l'Ouest-Africain, jusqu'au Sénégal. Sa famille linguistique appelée 'Niger-Congo', déjà suggérée par Mlle Lilias Homburger, élève d'Antoine Meillet, est un acquis fondamental : ici, les préjugés extra-scientifiques n'ont pas obnubilé le regard du savant américain. Tout n'est donc pas à rejeter dans la classification des langues africaines par Greenberg. $»^{24}$

Par ce compliment décerné in fine, T. Obenga croit peut-être se dédouaner des accusations inacceptables qu'il porte contre le même auteur en situant sa famille afroasiatique «dans ces catégories dictées par le racisme et bien périmées aujourd'hui $»^{25}$.

En fait, T. Obenga reprend quasiment telle quelle la disposition des phylums de Greenberg, à ceci près qu'il sort l'Égyptien, le Couchitique et le Tchadique de l'Afroasiatique pour les classer dans son Négro-Égyptien, aux côtés du Nilo-Saharien et du Niger-Kordofanien. Il laisse à part le Khoisan.

\section{Résurrection des fantômes}

T. Obenga s'est fait une spécialité de ranimer le vieux fantôme du chamitique (encore appelé « hamitique »), accusant les linguistes qui suivent en gros la classification de J.H. Greenberg d'être les suppôts de cette théorie. Il n'a manifestement pas bien compris que Greenberg, l'a justement radiée du domaine scientifique depuis près d'un demi-siècle.

T. Obenga confond également d'un même pas « chamitique » et " chamitosémitique ». Il faut avouer qu'il y a effectivement où s'y perdre, mais les spécialistes du niveau de T. Obenga savent tous que le terme de «chamitosémitique » ne recouvre pas d'un côté des langues "chamitiques", et de l'autres des langues « sémitiques ». On doit aussi remercier J.H. Greenberg d'avoir récusé cette dénomination qui prêtait à confusion, en la remplaçant par « Afroasiatique ».

Dernière chose. Les tenants de la thèse "chamitique // hamitique // afroasiatique » seraient mus par une idéologie raciste. Il serait bon de rappeler que, si les premiers tenants de la thèse «hamitique »-allemands, français ou autres - pratiquaient le type d'amalgame que l'on peut qualifier de « raciste" (considérations de couleurs de peau, de texture des cheveux, de modes de vie - nomade, de préférence -, de niveau intellectuel primitif, etc.), il ne faut pas jeter systématiquement l'opprobre sur tous ceux qui ont employé le terme, à l'époque. Certains l'ont employé dans un sens technique, leur seul tort étant de faire reposer cette pseudo-famille linguis-

\footnotetext{
${ }^{24}$ Ibid. p. 347.

${ }^{25} \mathrm{Ibid}$.
} 
tique sur des critères typologiques, et non sur des critères à la fois morphologiques et lexicaux.

\section{Ethnonymes et toponymes}

Pour tous les auteurs de la mouvance diopienne, ethnonymes et toponymes constituent une preuve irréfutable qui permet soit de définir le point de départ d'une migration, soit de jalonner un parcours migratoire. Voici quelques citations :

«[...] C'est surtout la toponymie, l'analyse des noms totémiques de clans que portent tous les Africains, soit à l'état collectif, soit à l'état individuel suivant le degré de dispersion, c'est l'analyse de ces noms associés à une analyse linguistique appropriée qui nous permettra de passer du plan de la probabilité au plan de la certitude » [sur la question du peuplement de l'Afrique à partir de la vallée du Nil]. ${ }^{26}$

«D'après de nombreuses traditions orales concordantes et que confirment de plus en plus les données archéologiques, linguistiques et ethnologiques, les nationalités et les peuples qui se sont succédé au Soudan central septentrional, à partir du $\mathrm{V}^{\text {ème }}$ siècle avant notre ère, comptent dans leur rang une foule de groupes Ngara, Nala, Kara, Tara, Djala, Wankara, Diala, ayant ceci en commun qu'ils se réclament du mouvement migratoire Changala - Changouls en arabe - ou Sangara, voire Sahara. En plus ils portent des noms similaires, sinon identiques. $\|{ }^{27}$

«Nous pensons que l'existence des noms totémiques typiques de l'ethnie haal-pulaar, à l'exclusion de toute autre, dans cette fameuse région des Collines de Nubie, montre amplement que l'ethnogenèse de ce groupe ne peut se situer dans la vallée du fleuve Sénégal. $»^{28}$.

Il suffit donc de trouver une concordance phonétique entre des toponymes, des ethnonymes ou des anthroponymes de l'est et de l'ouest (ou du centre) africains pour établir la preuve d'un contact ou d'une filiation historique.

L'un des pères de la linguistique historique moderne, Antoine Meillet, donne un avis circonstancié sur l'usage que l'on peut faire des noms propres :

«D'une manière générale, les étymologies de noms propres sont incertaines parce que, des deux données dont la concordance avec des faits d'autres langues établit la valeur, le sens et la forme phonique, on

\footnotetext{
${ }^{26}$ Diop 1965, p. 316.

${ }^{27}$ Dika Akwa 1985, p. 19.

${ }^{28}$ Lam 1993, p. 126.
} 
peut utiliser un seul : la forme phonique. Les linguistes qui s'intéressent surtout à l'étymologie des noms propres sont souvent des aventuriers de la linguistique [...]. . ${ }^{29}$

Il est pourtant évident que la toponymie, en particulier, peut être un auxiliaire précieux de l'historien. Cependant, le fait de retrouver deux toponymes identiques à des milliers de kilomètres l'un de l'autre, sous une forme quasiment identique, doit inciter à la prudence. Deux exemples vont situer les dangers d'une interprétation trop rapide.

On trouve près de La Mecque une petite colline du nom de Marwa, auprès de laquelle se rendent les musulmans qui font ce qu'il est convenu d'appeler le " petit pèlerinage ». Au Nord-Cameroun, d'autre part, on trouve Maroua [Marwa], une localité, investie par les Peuls dans les dernières années du XVIII ${ }^{\text {ème }}$ siècle. De plus, cette ville est adossée à des collines. Il serait tentant d'imaginer que la localité peule a été baptisée ainsi par des pèlerins retour de La Mecque. Cependant, il n'en est rien, et le nom de [Marwa] est l'adaptation à la phonologie peule du giziga [marva] " pierre de meule », qui fait référence à un événement historique du XVIII ${ }^{\text {ème }} \mathrm{s}$.

Dernier exemple. On connaît en Bretagne la localité de Carnac [Karnak], célèbre par ses monuments mégalithiques. Dans la vallée du Nil, on trouve le site de Karnak, entouré de hautes murailles de terre crue. Au plein coeur de l'Afrique centrale, on trouve, lorsque l'on consulte les vieilles cartes, la localité 'kotoko' de Logone ${ }^{30}$-Karnak. En fait, il n'y a aucune relation particulière entre ce toponyme breton et ses correspondants africains. Le nom breton se décompose en karn «tumulus de pierres » ( $c f$. irlandais cairn), suivi d'un suffixe $-a k$, dû à une influence romane. Quant aux Karnak d'Afrique, leur nom vient de l'arabe dialectal ${ }^{31}$ et signifie «forteresse » ou

\footnotetext{
${ }^{29}$ Meillet 1970, pp. 41-42.

${ }^{30}$ Une controverse se déroule actuellement sur le lieu de naissance de l'ancêtre africain du grand écrivain russe Pouchkine. L'une des thèses en présence veut que ce soit «Logone », ville de la rive camerounaise du fleuve Logone. On peut déjà préciser qu'il existe face à face deux «Logone», l'un étant donc sur la rive tchadienne. Cette localité est appelée «Loggun» dans le récit de voyage de Denham et Clapperton (1822-1824). Dans Sahara und Sudan, Nachtigal raconte son passage, en mars 1872, à «Logon», qu'il appelle aussi «Karnak» ou «Karnak Logon ». Ce n'est qu'ultérieurement que «Logone » apparaîtra sur les cartes. Pour les Allemands, le mot est prononcé en trois syllabes [logoné], et pour les Français, en deux [logon]. En fait, le véritable nom de la localité est [Lagwan]. C'est ainsi que le prononcent tous ses ressortissants. La prononciation [logoné] désigne les habitants de Lagwan.

${ }^{31}$ Carbou H., 1913, Méthode pratique pour l'étude de l'arabe parlé au Ouaday et à l'est du Tchad, Paris, Geuthner, p. 238.
} 
« village fortifié ». Il se trouve, effectivement, que Logone-Karnak (appelée de nos jours «Logone-Birni ${ }^{32}$ », est bien une ancienne cité fortifiée, et l'on peut encore voir des traces de ses remparts de terre.

On a ainsi montré, pense-t-on, que les toponymes ne parlent que si l'on a les moyens d'en faire l'étymologie. Oumar $\mathrm{Ba}$, mettant à profit cette méthode, a découvert d'anciens sites peuls en Mauritanie ${ }^{33}$. C. Gouffé (1967) et A. Kirk-Greene (1969) ont étudié soigneusement la toponymie haoussa. H.T. Sa'ad (1987) s'est intéressé au nom des villes peules de l'ancien Adamawa. Le point commun entre toutes ces études, c'est qu'elles reposent sur une étymologie précise, renvoyant à des langues contemporaines. Il est certes beaucoup plus difficile d'arriver à des résultats convaincants à partir de données décontextualisées et retenues pour leur seul aspect phonétique.

\section{La comparaison lexicale}

Le gros de l'argumentation de C.A. Diop et de ses émules repose sur la comparaison lexicale entre langues africaines et égyptien. L'égyptien se trouve hypostasié, et l'on oublie qu'il s'est développé sur une période de plusieurs millénaires, au cours desquels il a subi de profondes évolutions ${ }^{34}$, et été en contact avec de nombreuses autres langues, dont on doit penser que la plupart ont disparu.

De très nombreux biais entachent la validité générale ${ }^{35}$ des rapprochements faits par les diopiens entre égyptien et langues d'Afrique noire. Il semble que, pour eux, l'accumulation soit le principal argument probant, indépendamment de toute méthode rigoureuse. Nous allons donner quelques exemples des principales failles du dispositif.

\section{Sélection du vocabulaire}

Le vocabulaire dit «de base» est constitué des mots désignant les principales parties du corps (tête, oeil, langue, ventre, coeur, cheveux, oreille, bouche, pied, genou, etc.), les éléments naturels (eau, feu, soleil, lune, étoile, pierre, etc.), des actions du corps (manger, boire, dormir, sucer, sentir, marcher, voir, entendre, mourir, etc.)... Ce genre de termes est réputé moins susceptible d'être emprunté d'une langue à l'autre; lorsque l'on rencontre dans plusieurs langues, pour des mots de cet ensemble, des termes

\footnotetext{
${ }^{32}$ Cette fois, la deuxième partie du nom vient du kanuri [bərni], qui signifie « ville fortifiée $»$.

${ }^{33}$ Ba 1977, pp. 22-28.

${ }^{34}$ Loprieno 1995, pp. 5-8.

35 Rappelons que, si une bonne méthode garantit de bons résultats, une mauvaise peut, par hasard, et ponctuellement, mettre à jour des éléments intéressants.
} 
qui offrent une ressemblance phonétique, on peut raisonnablement en induire qu'ils proviennent d'un ancêtre commun.

Le vocabulaire technique doit faire l'objet d'une étude séparée, car ce n'est pas lui qui peut fonder en première analyse une parenté. En effet, il est susceptible de passer d'une langue à l'autre par le processus de l'emprunt. On le voit très facilement dans les langues africaines contemporaines du Sahel, par exemple, où une bonne partie du vocabulaire religieux et juridique vient de l'arabe. Il est donc plus difficile de savoir si ce type de vocabulaire est hérité ou emprunté.

C.A. Diop (1977) et Lam (1993) sont en constante contradiction avec ces principes. T. Obenga (1993), relativement à ces deux auteurs, peut être qualifié de rigoureux sur ce point.

\section{Rapprochements sémantiques contestables et création de chaînons sémantiques artificiels}

On ne fera pas le catalogue des rapprochements sémantiques étonnants contenus dans Diop et Lam, (ceux d'Obenga n'apparaissent pas dans ses listes comparatives finales, mais ils pullulent par ailleurs). Qu'il suffise d'en donner deux ou trois :

(a) égyptien ${ }^{36}: s h m . t$ 'divinité à tête de lion, chargée par dieu de détruire l'espèce humaine en engloutissant les individus dans sa gueule'; comparé avec le walaf ${ }^{37}$ : sahami 'mâcher'38.

(b) égyptien 'apporter, amener', comparé avec kanuri 'place, lieu' (là où l'on apporte); caffino 'esclave, serviteur' (celui qui apporte) ; mbosi 'chemin, route, direction' (moyen pour apporter) ; lètè 'pied' (marche : hiéroglyphe pharaonique $)^{39}$.

Un procédé astucieux, difficile à détecter par des locuteurs non natifs, consiste à inclure dans le sens d'un mot un élément qui lui est étranger, ou, au contraire (dernier exemple ci-dessous) d'en supprimer la valeur de base. En voici quelques exemples, pris au hasard - nous donnons en troisième position les données exactes - :

36 Dans les pages suivantes, nous citons toujours les formes égyptiennes telles qu'elles sont données respectivement par Diop, Obenga et Lam. Ici, en l'occurrence, c'est d'après Diop 1977.

${ }^{37}$ C'est sous cette forme que Diop désigne la langue généralement connue sous le nom de wolof.

${ }^{38}$ Diop 1977, p. 299.

${ }^{39}$ Obenga 1993, p. 199. 
(a) égyptien : $\underline{h} j j$ 'écorce de l'arbre comme cordon d'amulette' walaf $^{40}$ : heti 'découper une lanière très fine d'une écorce ou d'une peau' wolof $^{41}$ : xotti 1. 'déchirer' 2. 'acheter des vêtements ou un tissu' 3. 'licencier révoquer'.

(b) égyptien : $m n i$ 'agir comme un berger, un pâtre' walaf $^{42}: \min ($ al) 'domestiquer' wolof $^{43}$ : miin 'être habitué à, être familier à ; avoir l'habitude de voir'.

(c) égyptien : $\underline{h} 3 t$ 'cadavre'; $h 3 y t$ 'tas de cadavres walaf $^{44}$ : hèt 'odeur cadavérique ?' wolof ${ }^{45}$ : xet 'odeur'.

(d) égyptien : $h n$ 'coffre thoracique' pulaar $\mathrm{Lam}^{46}$ : galle 'cage, enceinte' pulaar Niang ${ }^{47}$ : galle 'concession, enclos familial; enclos pour animaux'. La valeur 'cage' n'existe pas pour le mot galle; cependant, par un enchaînement caché, via le français 'cage thoracique', elle permet de relier deux mots qui n'ont rien à voir ensemble.

(e) égyptien : $r$ 'bouche' pulaar Lam ${ }^{48}$ loow - 'mettre dans la bouche' pulaar Niang ${ }^{49}$ : loow- 'verser pour remplir ; remplir' Lam restreint abusivement le sens du pulaar.

(f) égyptien : $w 3 b w$ 'haut des cuisses' pulaar $\mathrm{Lam}^{50}:$ kab6i 'attaches' pulaar $^{51}$ : le radical verbal hab6- signifie 'attacher, lier'; la forme donnée par Lam est une forme plurielle conjuguée sans sujet ; en aucun

${ }^{40}$ Diop 1977, p. 261.

${ }^{41}$ Cité d'après Fal et alii, 1990, et Cissé 1998.

42 Diop 1977, p. 223.

${ }^{43} \mathrm{Fal}$ et alii, 1990, p. 133.

${ }^{44}$ Ibid. p. 283.

${ }^{45}$ Fal et alii, 1990, p. 256.

${ }^{46}$ Lam 1993, p. 380. Notons au passage le caractère improbable du rapprochement.

${ }^{47}$ Niang 1997, p. 35.

${ }^{48}$ Lam 1993, p. 380.

${ }^{49}$ Niang 1997, p. 61. Voir aussi Seydou 1998, p. 414.

${ }^{50}$ Lam 1993, p. 378.

${ }^{51}$ Seydou 1998, p. 241. 
cas le pulaar ne peut référer à une articulation, à la différence du français «attaches ».

(g) égyptien : $h f^{f}$ 'empoigner, capturer ; prendre, saisir fortement' fang $^{52}$ : kobe 'être armé' (se dresser, être prêt à frapper)

fang Galley ${ }^{53}$ : kobe '1. être au-dessus 2. se tendre, se dresser, être courbé, être armé (fusil, piège)' ; il est absolument clair qu'Obenga sollicite le sens de ce mot.

(h) égyptien : $b k 3$ 'être enceinte' ; copte boki 'concevoir, être enceinte' fang $^{54}: b i k \hat{\imath}$ 'dot de mariage' (même aire sémantique)

fang Galley ${ }^{55}$ : bikî, pl. de ékî, 'dot de mariage (nsua bikî). Une dot était composée autrefois de morceaux de fer. ékî, pl. bikî, 'fer, métal en général'.

Le long séjour gabonais d'Obenga et sa familiarité avec les civilisations bantoues auraient dû le prémunir contre une pareille erreur d'interprétation.

Un sommet est atteint dans l'imagination déployée pour relier entre eux des mots qui n'ont rien à voir ensemble, et dont, d'ailleurs, l'interprétation est controuvée. Nous citerons deux de ces passages d'anthologie :

1. [Obenga 1993, pp. 203-204 examine les mots négro-africains qu'il estime apparentés à l'égyptien sem (sm3) 'prêtre'. «Dans l'Égypte ancienne le prêtre sem (sm3) était vêtu d'une peau de panthère. Il est également connu qu'il avait une très haute fonction sacerdotale: la toilette divine, c'est-à-dire, pour la dogmatique pharaonique, l'entretien quotidien des forces divines qui assuraient ainsi l'ordre cosmique. »

[Suivent quelques citations de mots bantous et bambara pour 'sanctifier, honorer, bénir ; prier ; chef de culte ; propreté ; mots reposant tous sur le schème consonantique $s-m$. L'auteur passe ensuite à kanuri same 'ciel', hausa sama 'ciel', sénoufo sama 'bon', songhai sama 'être beau' et samaa 'conserver la santé, épargner la mort' ; bambara sama 'offrir un cadeau, faire présent'. Pour arriver à faire tenir ensemble tout cela ${ }^{56}$, voici la solution trouvée] :

\footnotetext{
52 Obenga 1993, p. 333.

53 Galley S., 1964, Dictionnaire fang-français et français-fang, H. Meisseiller, Neuchâtel, p. 155.

${ }^{54}$ Obenga 1993, p. 331.

${ }^{55}$ Galley S., 1964, pp. 69 et 99.

56 Nous avons déjà montré ailleurs en détail les manipulations sémantiques qu'Obenga fait subir ici aux langues qu'il cite. Voir Tourneux 1994, p. 157.
} 
«[...] le prêtre sem (sm3, sema), vêtu d'une peau de léopard lors de ses fonctions rituelles, sacerdotales, devait rendre le dieu habillé beau et fort, conserver ainsi sa santé ; les présents ne manquaient pas : étoffes, huiles, parfums, onguents, etc. Ainsi habillé, honoré, purifié, le dieu devait garder l'ordre cosmique, l'ordre céleste. [...] Les égyptologues [...] précisent que le prêtre [...] habillait plus spécialement les dieux Min, Horus, etc., c'est-à-dire les dieux éminemment protecteurs : Horus, c'est le grand dieu du ciel, le dieu royal par excellence, et Min, maître générateur, incarnait la divinité du «taureau qui couvre les femelles »: en baya [...] sami et samé signifie (sic) : « bélier ».

[La conclusion est à la hauteur de la démonstration :]

«Ce qui apparaît, à travers la linguistique proprement dite, c'est l'unité radicale de toutes les civilisations négro-africaines: les analyses sémantiques et culturelles qui précèdent le montrent suffisamment. On voit aussi qu'une véritable anthropologie culturelle de l'Afrique noire reste nécessairement suspendue en l'air tant que la Vallée du Nil, Égypte-Nubie-Abyssinie, n'est pas franchement sollicitée, prise en compte. Tout le reste est strictement banal sans l'intervention courageuse, mais appropriée, de l'égyptologie. On doit le dire avec netteté. »

2. [Lam 1993, pp. 234-235, tient à expliquer par le pulaar le nom égyptien de Hathor, la « déesse-vache ».]

«Hathor était qualifiée par les anciens Égyptiens de 'Dame de la joie, de la danse, de la musique, de l'amour, etc.' Elle incarnait donc la perfection de la grâce et de la sensualité. Cette manière de voir a été confirmée par le fait suivant : courroucé par les divergences intervenues entre les dieux à cause de la lutte entre Seth et Horus, Rê doit le retour de sa bonne humeur à Hathor. En effet, d'après Christiane DesrochesNoblecourt, 'c'est au cours de cet épisode que sa fille Hathor, pour le dérider, lui dévoila sa nudité'. C'est, nous semble-t-il, parce que Hathor avait un sexe attrayant du fait de sa perfection qu'elle a été promue dame de l'amour et qu'elle a réussi à ramener la joie chez Rê, le roi des dieux. Or, en pulaar, hottere signifie 'le grand sexe' (féminin) ${ }^{57}$. Il s'agit ici d'un augmentatif appliqué au terme kottu (sexe féminin) qu'on retrouve d'ailleurs en égyptien sous la forme $k 3 t$. Ce qui donne un mot composés $^{58}$ : hott-ere $>$ hottere qui est d'ailleurs parfaitement conforme à la structure du terme égyptien $h t-h r r$ (Hathor). Le pulaar nous permet de dévoiler totalement un aspect de la personnalité de la déesse égyptienne [...]. [...] la chose est indiscutable : hottere désigne bien le

\footnotetext{
${ }^{57}$ Ce dérivé, si tant est qu'il est attesté dans l'usage, ce dont il est permis de douter, ne pourrait pas avoir la valeur « augmentative » que lui attribue Lam.

${ }^{58}$ Ce procédé de formation des mots s'appelle « dérivation ».
} 
sexe parfait, idéal pourrait-on dire ; c'est sans doute pourquoi Hathor est arrivée à dérider Rê [...]. »

\section{Manipulations des données}

C.A. Diop, pour «aider» le lecteur à y voir plus clair, écrit le wolof selon les principes de la translittération de l'égyptien. M. Houis faisait part de son étonnement à ce sujet dans son article de 1980. On obtient alors des résultats de ce genre ${ }^{59}$ :

$\begin{array}{llllll}\text { égyptien } & \text { sens } & \text { walaf } & \text { sens } & \text { Fal 1990 } & \text { sens } \\ \text { idr } & \begin{array}{l}\text { troupeau } \\ \text { itt }\end{array} & \underline{\mathrm{dwr}} & \text { troupeau }^{61} & \text { jur } & \text { bétail } \\ \text { itt } & \text { s'envoler }^{\text {twt }} & \text { poussin }^{62} & \text { cuuj } & \text { poussin }\end{array}$

Pour augmenter le nombre de consonnes rapprochables entre égyptien et pulaar, Lam choisit souvent des formes non radicales des mots qu'il cite :

(a) égyptien wtr 'sang, vin' // pulaar wodeere 'rouge', wojju 'rouge' ${ }^{63}$ En fait, on a wodee- 'rouge' (suivi obligatoirement d'un des nombreux suffixes de classe de la langue); wojj- 'être rouge' est un radical verbal. Wodeere est arbitrairement accordé par Lam dans la classe nde; s'il se référait à un liquide, comme le sang, l'adjectif prendrait la forme mbodeejam, accordée dans la classe dam.

(b) égyptien mndt 'paupière' // pulaar majnata 'qui fait sourciller' ${ }^{\prime} 4$

En pulaar, on a un radical verbal maj-, suivi d'un factitif - $n$-, et d'un suffixe aspectuel -ata. Majnata est donc une forme conjuguée, sans sujet, que l'on peut traduire par «[ça] fait sourciller »; ce n'est absolument pas un participe comme le laisse entendre la traduction de Lam.

\section{Méconnaissance des emprunts}

Malgré le désir des uns et des autres de séparer définitivement toutes les langues «négro-égyptiennes » du sémitique, il est amusant de noter qu'ils n'hésitent généralement pas à rapprocher de l'égyptien des emprunts arabes présents dans ces mêmes langues négro-africaines :

\footnotetext{
${ }^{59}$ Diop 1977, p. 182-183.

${ }^{60}$ Troupeau de boeufs.

${ }^{61}$ Troupeau d'animaux domestiques.

62 On remarquera le hiatus sémantique entre l'égyptien et le wolof: le poussin est justement une forme d'oiseau qui ne peut pas s'envoler.

${ }^{63}$ Lam 1993, p. 378.

${ }^{64}$ Ibid. p. 379.
} 
(a) égyptien $m w y t$ 'urine' // pulaar bawle 'urine' ${ }^{65}$

En fait, le pulaar bawle vient de l'arabe baul 'urine' ${ }^{66}$.

(b) égyptien $d w 3$ 'prier' // pulaar duwaaw 'prière' ${ }^{97}$

La forme réelle du pulaar est duwaawu, 'prière, condoléances', et elle vient de l'arabe $d u^{c} \bar{a}$ ' 'appel ; invocation de Dieu, supplication, prière' ${ }^{968}$.

(c) égyptien imy- $r$ 'chef, responsable' // pulaar yamir- 'donner l'ordre' 69 Le pulaar yamir- vient de l'arabe 'amara 'ordonner, commander' ${ }^{70}$.

(d) égyptien sem 'prêtre' // kanuri same 'ciel', hausa sama ${ }^{71}$ Le kanuri $s a ́ m i i^{72}$ et le hausa samà 'ciel' viennent de l'arabe sama 'ciel'?3.

(e) égyptien sem 'prêtre' // songhai sama 'être beau'74

Le songhay sama vient de l'arabe sam h 'être généreux, aimable, etc. ${ }^{75}$, qui a donné, en arabe nigérian, par exemple, same, seme, 'bon, beau, agréable ${ }^{76}$.

\section{Non-respect de la classification dans l'établissement des rappro- chements}

T. Obenga, dont nous avons dit plus haut qu'il veillait à ne retenir que du vocabulaire de base pour ses comparaisons, annihile le bénéfice de cette procédure en négligeant constamment les acquis de la classification lorsqu'il donne ses listes comparatives ${ }^{77}$. Il cite, par exemple l'égyptien $f d w$

${ }^{65} \mathrm{Ibid}$. p. 379.

${ }^{66}$ Wehr H., 1979 (4 édition), A Dictionary of Modern Written Arabic, ed. by J.M. Cowan Wiesbaden, Harrassowitz, p.101.

${ }^{67}$ Lam 1993, p. 400.

${ }^{68}$ Wehr 1979, p. 327.

${ }^{69}$ Lam 1993, p. 401.

${ }^{70}$ Wehr 1979, p. 33. Voir Ba $1973^{2}$ et Seydou 1998.

${ }^{71}$ Obenga 1993, p. 203.

${ }^{72}$ Cyffer N. et Hutchison J., 1990, Dictionary of the Kanuri Language, Dordrecht / Foris, Maiduguri / University of Maiduguri, p.153.

${ }^{73}$ Wehr 1979, p. 504.

${ }^{74}$ Obenga 1993, p. 203.

${ }^{75}$ Wehr 1979, p. 499.

${ }^{76}$ Kaye A.S., 1986, Nigerian Arabic-English Dictionary, Malibu, Undena Publications, p. 70.

${ }^{77}$ Obenga 1993, pp. 283-342. 
« quatre $»^{78}$, qu'il rapproche de 42 termes « négro-africains » apparentés. Il conclut triomphalement: "C'est exactement le même lexème, depuis le vieil égyptien jusqu'aux langues négro-africaines modernes : aucun hiatus sur plus de 5000 ans. » Comme nous l'avons déjà dit ${ }^{79}$, au lieu de ces 42 termes, il aurait pu tout aussi bien en citer 150 ou 170, nombre approximatif des langues tchadiques contemporaines. En effet, il omet de dire que ces quarante-deux attestations valent pour une seule, toutes étant tirées exclusivement de la famille tchadique. Pour que sa démonstration soit probante, il aurait fallu que $f d w$ égyptien ait des correspondants non seulement en Tchadique, mais aussi en Nilo-Saharien, en Couchitique, en Omotique, en Niger-Kordofanien, ce qui n'est pas le cas.

Pour valider le rapprochement entre une langue donnée et l'égyptien ancien, $\mathrm{T}$. Obenga propose une méthodologie qui fait fi de toutes les pratiques scientifiques :

«[...] la coïncidence de trois langues non contigües suffit donc pratiquement à garantir le caractère commun, 'négro-égyptien', d'un mot. C'està-dire si je compare l'égyptien et le mbochi, je dois nécessairement apporter le témoignage au moins d'une langue, aussi bien éloignée, dans l'espace, de l'égyptien (Vallée du Nil) que du mbochi (cuvette congolaise). »

Il est bien évident que l'éloignement géographique ne signifie absolument rien en ce domaine. Le mbosi et le wolof, situés à des milliers de kilomètres de distance géographique, sont proches génétiquement parlant, étant membres de la même famille Niger-Congo, au sein du phylum NigerKordofanien. En revanche, le hausa et le kanuri, juxtaposés sur le terrain, sont éloignés au maximum d'un point de vue génétique, le premier appartenant à la famille Tchadique, au sein du phylum Afroasiatique, le deuxième appartenant à la famille du Saharien, au sein du phylum NiloSaharien.

Par ailleurs, dans sa volonté de couper tout lien de parenté possible entre l'Égyptien et le Sémitique, Obenga dissimule au lecteur des faits très connus de tous les comparatistes. C'est le cas de l'égyptien $m t, m w t$ « mourir », dont on connaît depuis longtemps la parenté avec le sémitique $m w t^{80}$.

\footnotetext{
${ }^{78}$ Ibid. pp. 323-324.

79 Tourneux 1994, p. 156.

${ }^{80}$ Cohen 1969, p. 192, n 488.
} 


\section{Confusion entre classification et reconstruction}

En conclusion à son ouvrage principal consacré à la question linguistique, $\mathrm{T}$. Obenga écrit ceci :

«[...] nous avons pu reconstruire le négro-égyptien, soit la langue primitive commune aux langues historiquement attestées que sont les langues égyptienne, couchitique, tchadique, nilo-saharienne, nigérokordofanienne, toutes les langues anciennes et modernes, parlées par les peuples noirs d'Afrique, depuis la vieille Egypte pharaonique, et toutes unies génétiquement lorsqu'on compare de façon serrée et adéquate sous tous les angles, phonétique, phonologique, morphologique, grammatical, lexicologique. $»^{81}$

En réalité, on ne trouve aucune trace de reconstruction dans tout le livre. On peut en déduire, soit que l'auteur ignore ce qu'est la reconstruction, ce qui est difficile à croire, soit qu'il confond classification et reconstruction, péché mignon des indo-européanistes justement dénoncé par Ruhlen (1997), soit encore que, pour lui, l'égyptien est la langue négro-africaine commune (inutile de reconstruire ce qui est donné), le terme de " négro-égyptien », qui laisse supposer une langue commune antérieure à la séparation entre égyptien et langues africaines n'étant alors qu'un artefact.

\section{Souhait final}

T. Obenga, qui se présente justement comme le principal continuateur de C.A. Diop ${ }^{82}$ considère l'égyptien comme le sanskrit de l'Afrique ${ }^{83}$. Il reste à espérer que son négro-égyptien n'en devienne pas l'indo-germanique.

Heureusement, la recherche linguistique se poursuit. Pour certains, la place de l'ancien égyptien au sein de l'Afroasiatique n'est pas assurée et les rapprochement faits entre le sémitique et l'égyptien pourraient s'expliquer par des contacts ${ }^{84}$. Pour d'autres, cependant,

«Earlier Egyptian exhibits four sets of personal pronouns, which share many elements with the pronoun of other Afroasiatic languages [...]. $\rangle^{85}$

\footnotetext{
${ }^{81}$ Obenga 1993, p. 373.

${ }^{82}$ Ibid. pp. 10, 373.

${ }^{83}$ Ibid. p. 8.

${ }^{84}$ Bechhaus-Gerst 1998.

85 Loprieno 1995, pp. 63-67. «L'égyptien primitif possède quatre ensembles de pronoms personnels, qui ont de nombreux éléments communs avec le pronom d'autres langues afroasiatiques [...]. »
} 
Et l'on sait qu'il est difficile de supposer qu'un tel système s'emprunte d'une langue à une autre...

On continue à explorer les relations entre phylums au sein de la classification de Greenberg. L'hétérogénéité constatée au sein du Nilo-Saharien, par exemple, laisse supposer que la langue commune, si elle a existé, est de très loin antérieure à celle du Niger-Kordofanien. La famille khoisan représente peut-être le dernier témoignage des langues aborigènes d'Afrique. Etc.

La réflexion théorique sur les notions de filiation généalogique, de tradition et de constitution de langue s'approfondit. G. Manessy, dans un article bref, mais passionnant ${ }^{86}$, décrit typologiquement les huit cas de figures possibles de constitution de langues. Ce modèle repose sur la combinaison de trois éléments indépendants: le code linguistique, les automatismes physiologiques et cognitifs, et les représentations. Il appelle ' cryptotypes' " des schémas culturellement définis [...] qui caractérisent, au même titre que les techniques du corps, des aires de civilisations $»^{87}$. Le lien que l'on trouverait de manière récurrente, par exemple, entre «manger», «main droite» et «côté droit ${ }^{88}$, indépendamment des codes linguistiques dans lesquels il pourrait s'exprimer, constituerait l'un de ces cryptotypes constitutifs d'une aire culturelle donnée.

Les comparatistes qui se lancent dans ce qu'il est convenu d'appeler des «comparaisons à longue distance» doivent veiller à la sélection du vocabulaire de base sur lequel reposera leur démonstration ${ }^{89}$. Ils doivent également se prémunir contre des rapprochements sémantiques ad hoc. R. Boyd $^{90}$, examinant les principes adoptés par Greenberg dans sa méthode comparative, écrit ceci :

«Les principes que Greenberg applique pour autoriser des corrélats de sens non identiques sont simples et peu nombreux. On peut accepter :

1. des termes traduits par des quasi-synonymes en anglais: 'rope, string', 'ask, beg', ou par des termes qui se recouvrent largement d'un point de vue sémantique, et qui sont souvent exprimés par des

\footnotetext{
${ }^{86}$ Manessy 1992.

${ }^{87}$ Ibid., p. 72.

${ }^{88}$ Lam a établi la relation sur ce point entre le pulaar et l'égyptien. En swahili, on a de même mkono wa kulia, litt. 'la main pour manger' pour signifier "main droite». Il faudrait voir si cette pratique culturelle de manger avec la main droite exclusivement, déborde de l'aire d'influence de l'islam en Afrique noire.

${ }^{89}$ Boyd 1996, pp. 31-33.

${ }^{90}$ Ibid., p. 31.
} 
termes uniques dans les langues africaines: 'souhaiter, permettre', 'bon, beau', 'long, haut, profond, loin', 'huile, graisse' ;

2. des termes impliqués et coordonnés: 'tuer, mourir', 'acheter, vendre' (les couples de ce genre sont en petit nombre)

3. des noms et les verbes qui expriment l'action ou la production des mêmes noms : 'feu, brûler', 'lune, briller', 'hôte, rendre visite', 'noeud, lier', 'urine, uriner' ;

4. des relations métonymiques: 'sang, rouge', 'oiseau, poulet', 'pou, puce', 'visage, oeil', 'arc, flèche', 'verser, jeter', 'prendre, dérober'.

Les cas 2-4 concernent des termes qui, le plus souvent, sont exprimés par des mots différents dans chaque langue individuelle [...]. Les exemples 1-3 ne posent généralement pas de problèmes ; ceux du $n^{\circ} 4$, cependant, laissent place au doute. [...] Pour éviter une critique inutile, il serait sage d'attester l'existence de telles relations [métonymiques] en Afrique, en citant des exemples pris dans des langues contemporaines qui emploient de tels termes avec un certain chevauchement sémantique, ou dans des groupes dialectaux où l'on peut être raisonnablement certain que de tels changements ont réellement eu lieu dans une période récente. $»^{91}$

${ }^{91}$ Ibid. p. 31, trad. de nous. 


\section{Annexe}

Le tableau qui suit a été élaborée ${ }^{92}$ en adoptant des latitudes analogues à celles que l'on peut trouver dans les études linguistiques « négro-égyptiennes », tant en ce qui concerne les rapprochements phonétiques que sémantiques. Le résultat est troublant, et le lecteur pourra en conclure, au choix :

1. que le wolof vient de l'anglais;

2. que l'anglais vient du wolof;

3. que l'anglais a emprunté une partie de son lexique aux langues celtiques, qui elles-mêmes l'ont pris au wolof ${ }^{93}$;

4. que le wolof a emprunté une partie de son lexique à l'anglais ${ }^{94}$, via les langues brittonniques ;

5. que l'anglais et le wolof ont un ancêtre commun ${ }^{95}$;

6. que tout cela n'a aucun sens.

Pour parfaire la démonstration, nous pouvons établir les correspondances phonétiques suivantes ${ }^{96}$ :

\begin{tabular}{|c|c|c|c|}
\hline wolof & anglais & wolof & anglais \\
\hline p- & b- & c- & ch- \\
\hline b- & $\mathrm{b}-/ \mathrm{m}-$ & j- & j- \\
\hline f- & f- & $\mathrm{k}-$ & $\mathrm{k}-/ \mathrm{c}-$ \\
\hline $\mathrm{t}-$ & $\mathrm{t}-$ & g- & g- \\
\hline d- & d- & m- & $\mathrm{m}-/ \mathrm{w}-$ \\
\hline d- & -th & n- & n- \\
\hline -ay- & -i- & $1-$ & 1- \\
\hline
\end{tabular}

${ }^{92}$ En une heure, ce qui est une preuve de la validité de la méthode, si l'on en croit Ruhlen 1997, p. 156.

${ }^{93}$ Evidemment avant que les Proto-Celtes ne quittent la vallée du Nil ; voir Ahmed Ali et Ibrahim Ali, 1995, Pre-Celtic Languages, The African Substratum Theory, Punite Books, Cardiff, $142 \mathrm{p}$.

${ }^{94}$ L'emprunt a pu se faire suite au retour en Grande-Bretagne d'esclaves celtes, emmenés en Amérique par des navigateurs scandinaves (Vikings) vers la fin du premier millénaire, et ayant fait une escale prolongée en Afrique occidentale lors de leur retour. Voir Encyclopaedia Universalis, article « Navigation (Histoire de la) $\gg$.

${ }^{95}$ Pour étayer cette hypothèse, nous disposons de l'ethnonyme celte Dumnonii, qui a donné le moderne «Devon » en Cornouaille britannique, et qui signifie étymologiquement «les sombres »- presque noirs. Voir Sergent 1995, p. 209.

${ }^{96}$ Rappelons quand même qu'une correspondance phonétique n'est pas une preuve de parenté. Revoir Ruhlen, ci-dessus. 
Essai de comparaison langue à langue entre le wolof et l'anglais

\begin{tabular}{|c|c|c|c|}
\hline $\operatorname{sens}$ & wolof & anglais & sens \\
\hline anus & rasu & ass & cul \\
\hline apercevoir & séen & seen & $v u$ \\
\hline bouchée & lump & lump & $\begin{array}{l}\text { morceau (de sucre que l'on } \\
\text { peut engloutir en une seule } \\
\text { bouchée) }\end{array}$ \\
\hline bout du sein & cus & chew & mâcher \\
\hline chiot & kuti & kitty & chaton \\
\hline crasseux (être) & dërkiis & dirty & sale \\
\hline cuir & der & (lea)ther & cuir \\
\hline entasser & somm & sum & total \\
\hline éponger & màpp & wipe & essuyer \\
\hline être abondant & bari & many & nombreux \\
\hline être habitué & tàmm & tame & apprivoisé \\
\hline fatigué (être) & tàyyi & tired & fatigué \\
\hline fête & gàmmu & game & jeu \\
\hline grand-mère & maam & mummy & grand-mère \\
\hline hacher & dogat & cut & couper \\
\hline haricot & ñebbe & bean & haricot (métathèse) \\
\hline harpon & kajj & catch & attraper \\
\hline haut & kaw & high & haut \\
\hline jambe & tànk & leg & jambe \\
\hline lavoir & póotu & boat & bateau (cf. bateau-lavoir) \\
\hline lion & gaynde & lion & lion \\
\hline malaxer & jamb & jam & confiture (de fruits malaxés) \\
\hline mettre en petits tas & saam & sample & échantillonner \\
\hline mourir & dee & die & mourir \\
\hline mourir, décéder & fàddu & fade & s'effacer \\
\hline néant & neen & none & aucun \\
\hline ovinés & gàtt & goat & chèvre \\
\hline percer le nez & nos & nose & $n e z$ \\
\hline plat de riz en sauce & $\mathrm{cu}$ & stew & ragout \\
\hline porter (sur le dos) & boot & bore & supporter (preterit) \\
\hline prélever une partie su- & coppati & chop & couper à la hache \\
\hline perficielle & & chip & copeau \\
\hline puce & fel & flea & puce \\
\hline $\begin{array}{l}\text { puiser (un liquide dans } \\
\text { un récipient) }\end{array}$ & $\operatorname{tanq}$ & tank & réservoir \\
\hline rester & des & stay & rester (métathèse) \\
\hline s'abriter de la pluie & seelu & ceiling & plafond \\
\hline s'emparer de & siif & thief & voler \\
\hline sonder & cuq & check & vérifier \\
\hline
\end{tabular}




\section{Bibliographie}

Anselin, Alain, 1981, La question peule et l'histoire des Egyptes ouestafricaines, Paris, Karthala.

Ba, Oumar, 1973¹, Glossaire de mots mandé passés en poulâr du Foûta Tôro, Bulletin de l'IFAN, T. XXXV, série B, n 2, pp. 433-443.

Ba, Oumar, $1973^{2}$, Glossaire de mots étrangers passés en poulâr du Foûta Tôro, Bulletin de l'IFAN, T. XXXV, série B, n 3, pp. 676-711.

Ba, Oumar, (1977), Le Foûta Tôro au carrefour des cultures, Paris, Librairie-Editions L'Harmattan, 426 p. [Reprend entre autres les deux articles de 1973.]

Bechhaus-Gerst, Marianne, 1998, Old Egyptian and Afroasiatic, The State of the Art, Afrikanistische Arbeitspapiere 56, Cologne (RFA), pp. 111-129.

Bouquiaux, Luc, 1996, L'Afrique en quête de ses ancêtres. Réflexions d'un linguiste sur l'idéologie afrocentriste de Cheikh-Anta Diop et Théophile Obenga, Frankfurter Afrikanistische Blätter 8, pp. 135143.

Boyd, Raymond, 1996, Congo-Saharan revisited, Frankfurter Afrikanistische Blätter 8, pp. 15-48.

Brucker, Charles, 1988, L'étymologie, coll. «Que sais-je?», Paris, PUF, $125 \mathrm{p}$.

Cissé, Mamadou, 1998, Dictionnaire français-wolof, Paris, L'Asiathèque, $381 \mathrm{p}$.

Cohen, Marcel, 1969, Essai comparatif sur le vocabulaire et la phonétique du chamito-sémitique, Paris, Honoré Champion, XI + 248 p.

Diagne, Pathé, 1984, Introduction au débat sur les ethnonymes et les toponymes, in UNESCO, 1984, Ethnonymes et toponymes africains, Paris, UNESCO, pp. 11-17.

Diakonoff, I.M., 1988, Afrasian Languages, Moscou, Nauka, 144 p.

Dika Akwa nya Bonambela, Prince, 1982, Problèmes de l'anthropologie et de l'Histoire africaines, Yaoundé, Clé, $372 \mathrm{p}$.

Dika Akwa nya Bonambela, Prince, (1985), Les descendants des pharaons à travers l'Afrique, s. 1. (imprimé en Belgique, Bruxelles), Editions Osiris-Africa, $440 \mathrm{p}$.

Diop, Cheikh Anta, 1959, L'unité culturelle de l'Afrique noire. Domaines du patriarcat et du matriarcat dans l'antiquité classique, Paris, Présence Africaine, $203 \mathrm{p}$.

Diop, Cheikh Anta, 1960, Les fondements culturels techniques et industriels d'un futur État fédéral d'Afrique noire, Paris, Présence Africaine, $114 \mathrm{p}$. 
Diop, Cheikh Anta, 1962'1, Histoire primitive de l'humanité : évolution du monde noir, Bulletin de l'IFAN, T. XXIV, série B, n 3-4, pp. 449541.

Diop, Cheikh Anta, 1962², Réponses à quelques critiques, Bulletin de l'IFAN, T. XXIV, série B, n 3-4, pp. 542-574.

Diop, Cheikh Anta, 1965 (2 $2^{\text {ème }}$ éd.), Nations nègres et culture, Paris, Présence Africaine, 532 p. [1 ${ }^{\text {ère }}$ éd. 1955.]

Diop, Cheikh Anta, 1967, Antériorité des civilisations nègres: mythe ou vérité historique?, Paris, Présence Africaine, 299 p., 89 pl. hors texte.

Diop, Cheikh Anta, 1973, Introduction à l'étude des migrations en Afrique centrale et occidentale. Identification du berceau nilotique du peuple sénégalais, Bulletin de l'IFAN, T. XXXV, série $\mathrm{B}, \mathrm{n}^{\circ} 4$, p. 769-792.

Diop, Cheikh Anta, 1977, Parenté génétique de l'égyptien pharaonique et des langues négro-africaines. Processus de sémitisation, Dakar, Les Nouvelles Editions africaines / IFAN, XXXVII + 402 p.

Diop, Cheikh Anta, 1984, Pour une méthodologie de l'étude des migrations, in UNESCO, 1984, Ethnonymes et toponymes africains, Paris, UNESCO, pp. 97-121.

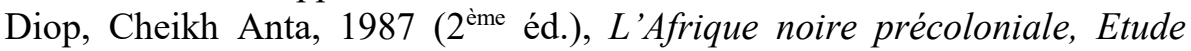
comparée des systèmes politiques et sociaux de l'Europe et de l'Afrique noire, de l'Antiquité à la formation des Etats modernes, Paris-Dakar, Présence Africaine, 278 p. (1 ${ }^{\text {ère }}$ éd. 1960).

Diop, Cheikh Anta, 1988, Nouvelles recherches sur l'égyptien ancien et les langues négro-africaines modernes. Compléments à "Parenté génétique de l'égyptien pharaonique et des langues négroafricaines », Paris-Dakar, Présence Africaine, 221 p.

El Fasi, Mohammed, 1984, La toponymie et l'ethnonymie sciences auxiliaires de l'histoire, in UNESCO, 1984, Ethnonymes et toponymes africains, Paris, UNESCO, pp.19-23.

Fal, Arame, Santos, Rosine, Doneux, Jean Léonce, 1990, Dictionnaire wolof-français, suivi d'un index français-wolof, Paris, Karthala, $342 \mathrm{p}$.

Fauvelle, François-Xavier, 1996, L'Afrique de Cheikh Anta Diop. Histoire et idéologie, Préface d'Elikia M'Bokolo, Paris, Karthala et Centre de recherches africaines, $237 \mathrm{p}$.

Gouffé, Claude, 1967, Problèmes de toponymie haoussa. Les noms de villages de la région de Maradi, Revue internationale d'Onomastique, pp. 95-127.

Greenberg, Joseph H. 1955. Internal a-plurals in Afroasiatic (HamitoSemitic). In Afrikanistische Studien [Festschrift Westermann], éd. par Johannes Lukas, Berlin, pp. 198-204. 
Greenberg, Joseph H., 1970 (3 $3^{\text {ème }}$ éd.), The Languages of Africa, Bloomington, Indiana University; The Hague, Mouton and Co, VII $+180 \mathrm{p}$.

Gregersen, Edgar E., 1972, Kongo-Saharan, Journal of African Languages, 11(1), pp. 69-89.

Houis, Maurice, 1980, Egyptien pharaonique et langues africaines, Afrique et Langage 13, pp. 69-79.

Kirk-Greene, Antony, 1969, The meaning of place names in Hausaland, Bulletin de l'IFAN, T. XXXI, série B, $\mathrm{n}^{\circ}$ 1, pp. 264-278.

Lam, Aboubacry Moussa, (1993) De l'origine égyptienne des Peuls, Paris, Présence Africaine; Gif-sur-Yvette, Khepera, 464 p., planches hors texte.

Lam, Aboubacry Moussa, 1983, Le pulaar viendrait-il de la vallée du Nil ?, Bulletin de l'IFAN, T. 45, série B, n 1-2, pp. 197-205.

Lam, Aboubacry Moussa, (1997) Les chemins du Nil. Les relations entre l'Egypte ancienne et l'Afrique noire, Paris, Présence Africaine; Gif-sur-Yvette, Khepera, 223 p.

Leslau, Wolf, 1962, A Prefix h in Egyptian, Modern South Arabian, and Hausa, Africa XXXII(1), pp. 65-68.

Loprieno, Antonio, 1995, Ancient Egyptian, A linguistic introduction, Cambridge / New York / Melbourne, Cambridge University Press, $\mathrm{XV}+322 \mathrm{p}$.

Manessy, Gabriel, 1992, Généalogie et génétique, Linguistique Africaine 9, pp. 67-75.

Meillet, Antoine, 1970, La méthode comparative en linguistique historique, Paris, Honoré Champion, VII + 117 p. (1 ère éd. 1925).

Meillet, Antoine, 1975, Linguistique historique et linguistique générale, Paris, Honoré Champion, 335 p.

Newman, Paul, 1995, On being right. Greenberg's African linguistic classification and the methodological principles which underlie it, Institute for the Study of Nigerian Languages and Cultures, Bloomington, Indiana University, $25 \mathrm{p}$.

Niang, Mamadou, 1997, Pulaar-English / English-Pulaar, (Hippocrene Standard Dictionary), New York, Hippocrene Books, XXIV p. + $276 \mathrm{p}$.

Obenga, Théophile, 1993, Origine commune de l'égyptien ancien, du copte et des langues négro-africaines, Introduction à la linguistique historique africaine, Paris, L'Harmattan, $402 \mathrm{p}$.

Obenga, Théophile, 1998, Les derniers remparts de l'africanisme, Présence Africaine 157, pp. 47-65.

Rostaing, Charles, 1985 (10 ${ }^{\text {ème }}$ éd.), Les noms de lieux, coll. "Que saisje ?», Paris, PUF, $128 \mathrm{p}$.

Ruhlen, Merritt, 1997, L'origine des langues, s.1., Belin, 288 p. 
Sa'ad, Hamman Tukur, 1987, Reflections on Fulfulde toponymy : a study of Fulbe towns in old Adamawa emirate, Annals of Bornu IV, pp. 724.

Sergent, Bernard, 1995, Les Indo-Européens, Histoire, langues, mythes, Paris, Payot \& Rivages, $536 \mathrm{p}$.

Seydou, Christiane, 1998, Dictionnaire pluridialectal des racines verbales du peul, peul-français-anglais; A Dictionary of Verb Roots in Fulfulde Dialects, Fulfulde-French-English, avec la collaboration de D.W. Arnott, H. Bocquené, F. Fagerberg-Diallo, F.S. Kâ, M. McIntosh, O. Ndoudi, A.M. Yattara, Paris, Karthala-ACCT, LIII + $896 \mathrm{p}$.

Tourneux Henry, 1994, Les langues africaines et l'égyptien, Politique africaine, 55, pp. 153-158.

UNESCO, 1984, Ethnonymes et toponymes africains, Histoire générale de l'Afrique, Etudes et documents 6, Paris, UNESCO, 208 p.

Zaborski, Andrzej, 1986, The Morphology of Nominal Plural in the Cushitic Languages, Beiträge zur Afrikanistik, Vienne, Afro-Pub, II + $307 \mathrm{p}$. 